\title{
THE STUDY OF SHIP COLLISION AVOIDANCE ROUTE PLANNING BY ANT COLONY ALGORITHM
}

\author{
Ming-Cheng Tsou \\ Department of Shipping Technology, National Kaohsiung Marine University, Kaohsiung, Taiwan, R.O.C, \\ d86228006@yahoo.com.tw \\ Chao-Kuang Hsueh \\ Department of Merchant Marine, National Taiwan Ocean University, Keelung, Taiwan, R.O.C
}

Follow this and additional works at: https://jmstt.ntou.edu.tw/journal

Part of the Transportation Engineering Commons

\section{Recommended Citation}

Tsou, Ming-Cheng and Hsueh, Chao-Kuang (2010) "THE STUDY OF SHIP COLLISION AVOIDANCE ROUTE PLANNING BY ANT COLONY ALGORITHM," Journal of Marine Science and Technology. Vol. 18: Iss. 5, Article 16.

DOI: $10.51400 / 2709-6998.1929$

Available at: https://jmstt.ntou.edu.tw/journal/vol18/iss5/16

This Research Article is brought to you for free and open access by Journal of Marine Science and Technology. It has been accepted for inclusion in Journal of Marine Science and Technology by an authorized editor of Journal of Marine Science and Technology. 


\section{THE STUDY OF SHIP COLLISION AVOIDANCE ROUTE PLANNING BY ANT COLONY ALGORITHM}

Acknowledgements

It is appreciated that this research is subsidized by funding from the National Taiwan Ocean University (NTOU-RD972- 01-03-34-01). 


\title{
THE STUDY OF SHIP COLLISION AVOIDANCE ROUTE PLANNING BY ANT COLONY ALGORITHM
}

\author{
Ming-Cheng Tsou* and Chao-Kuang Hsueh**
}

Key words: ant colony algorithm, collision avoidance, GIS, e-navigation.

\begin{abstract}
Maritime traffic is becoming more complex every day. At present, due to technological advances and to new maritime regulations, there is increasing demand for new nautical marine instruments to be installed into the bridge, and the breadth of navigational information complicates on-duty officers' decisions. Therefore, if decision support tools can be used to help deal with navigational decision-making, human errors arising from subjective judgments can be reduced, and sea transport safety improved. This research uses the concept of e-navigation as a framework, positioning collision avoidance path planning as the main theme, and applies an Ant Colony Algorithm (ACA) in the field of artificial intelligence to construct a collision avoidance model that imitates optimization behaviors in real-life applications. This model combines navigational practices, a maritime laws/regulations knowledge base and real-time navigation information from the AIS to plan a safe and economical collision avoidance path. Through using such planning, recommendations can be made for collision avoidance and return to course. Lastly, a Geographic Information System (GIS) was used as the platform for a navigation decision support system, combining related navigation information, collision avoidance models and electronic charts. This is a source of reference for VTS (Vessel Traffic Service) operators and on-duty officers to assess collisions in territorial waters, achieving objectives such as warning and pre-collision preparations.
\end{abstract}

\section{INTRODUCTION}

Ship collision avoidance is an important research domain in maritime science. Over the decades, scholars and experts have actively researched strategies for collision avoidance. This is

Paper submitted 04/09/09; revised 09/15/09; accepted 10/19/09. Author for correspondence: Ming-Cheng Tsou (e-mail:d86228006@yahoo.com.tw).

*Department of Shipping Technology, National Kaohsiung Marine University, Kaohsiung, Taiwan, R.O.C.

**Department of Merchant Marine, National Taiwan Ocean University, Keelung, Taiwan, R.O.C. because accidents such as ship collisions or groundings frequently happen, due to factors relating to the territorial waters navigated in, wave and weather conditions, traffic density, condition of ship body and navigator experience/skill. This can seriously threaten staff safety, result in financial loss and affect the marine environment. According to statistical analysis, incidents of ship collisions at sea are $80 \%$ due to human factors [15]. This indicates failure in and inaccuracy of navigator assessments with respect to ship movement, collision avoidance timing, collision danger estimation and appropriate avoidance strategies. Therefore, researching an automated decision making system for ship collision avoidance can increase the safety and reliability of a ship's automated navigation, reduce the psychological and physical burden of navigators, and reduce the occurrence of ship collisions, groundings and other sea accidents. Furthermore, at present, due to technological advances and the promulgation of new maritime regulations, various new types of nautical instruments are increasingly being installed into the bridge. This can result in navigational information overload, which can in turn adversely affect a navigator with insufficient experience when making decisions, resulting in crude or incorrect decisions that incur enormous costs. Therefore, by making the ship more intelligent and navigation more automated through technology (so as to reduce manual operations and the amount of subjective decision making), a navigator's burden is reduced and ship collision avoidance becomes more automated/intelligent. This is an effective method to solve human-related problems. For this purpose, in 2005, IMO (the International Maritime Organization) has advocated the e-navigation concept [18]. Under this concept, onboard navigation systems will be developed that utilize the integration of own ship sensors, supporting information, a standard user interface and a comprehensive system for managing guard zones and alerts. Core elements of such a system will include features such as high integrity electronic positioning, Electronic Navigational Charts (ENC) and analysis capability to reduce human error, whilst actively engaging the mariner in the process of navigation, at the same time preventing distraction and overburdening [1]. With e-navigation, apart from being used to assist officers on board, the other concept is to strengthen the shore-based VTS function. It can also be used for the management of vessel traffic, while related services from ashore will be enhanced through better provisioning, coordination and exchange of com- 
prehensive data in formats that will be more easily understood and utilized by shore-based VTS operators, in support of vessel safety and efficiency [2].

Targeting the realization of e-navigation, from the perspective of information analysis, ship collision avoidance can be regarded as a complex decision making process. It concerns the interaction between major factors such as the environment, ships and humans, dynamic and static data, known and uncertain information, quantitative mathematical calculations and qualitative logical reasoning, etc. It is also related to disciplines such as marine science and computer science. Whether it be a matter of information collection, information preprocessing, calculation of the degree of collision danger, determination of situation encountered, selection of avoidance method, optimization of collision avoidance motion, return navigation strategy or multi-target avoidance, it forms a complicated system engineering problem [13]. It is unrealistic to use an accurate mathematical model to describe such a system and apply it in a real-time decision making environment. Apart from this, collision avoidance is a multi-criteria, nonlinear programming problem, and a balance between navigational safety and economy needs to be achieved at the same time [19]. Also, apart from ensuring avoidance procedures that are necessary to ensure navigational safety, it must also be ensured that the avoidance route does not deviate too much from the original route, in order not to overly incur additional navigation costs. Therefore, in recent years, researchers have begun to use various artificial intelligence techniques to solve collision avoidance problems, whereby neural networks, fuzzy logic and evolutionary computing (in contrast to pure mathematical models for soft computing) address the collision avoidance problem [20].

From the perspective of information processing and integration, Automatic Radar Plotting Aid (ARPA) has always played an important role to assist in solving collision avoidance problems. Although it integrates related navigation information and in effect solves the information processing problem in the collision avoidance process, as well as providing fast and detailed, related collision avoidance information, it does not suggest collision avoidance or automated avoidance. It ultimately has to rely on the navigator's experience and his/her expert but subjective decision, and this can result in careless use and the creating of serious errors [4]. Recently, with the application of the Automatic Identification System (AIS) in ship collision avoidance and VTS, the problem of extracting real-time information for collision avoidance decision making has been resolved. The AIS system provides previously unavailable static information and accurate real-time dynamic information about the ship. This forms an important information source for collision avoidance decision making, beneficial for improving current collision avoidance methods and port traffic management. Therefore, this research uses the e-navigation initiative as a framework, and the Ant Colony Algorithm from artificial intelligence to create a collision avoidance model that imitates optimization behaviors in real-

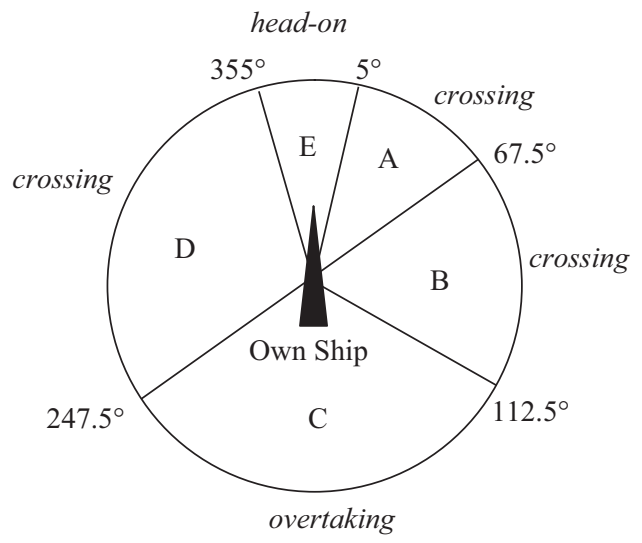

Fig. 1. Chart divisions show states encountered by ship.

life applications. Furthermore, through using GIS as platform, it combines navigational practice and a maritime laws knowledge base as well as real-time navigation information from AIS to construct a navigation decision support system and help plan a suitable collision avoidance path. This path would simultaneously observe economic and safety matters, as the shortest collision avoidance route on the safety critical is searched for. Through such planning, collision avoidance is suggested and restoration measure is also provided. At the same time, this would serve as a reference for VTS (Vessel Traffic Service) operators and ship on-duty officers for assessing collision avoidance in present conditions, achieving objectives such as warning and preliminary collision avoidance preparation.

\section{ASSESSMENT OF COLLISION RISK AND ACTIONS}

Our approach to assessing the collision risk is based on using a knowledge-based system. The knowledge-based system embodies collision avoidance techniques by using the 1972 International rules for collision avoidance (COLREGS) as the key information. The calculations for collision avoidance path planning are only executed when the collision avoidance conditions in the knowledge base have been satisfied. According to the combined results from analysis of COLREGS, navigation practices and automated collision avoidance methods, the encounter situation covered by COLREGS is divided into three types, where each type in turn is divided into subdivisions. The three main types of encounter situation are discussed below:

(1) Head-On: target ship approaching from E region in Fig. 1. The own ship and target ship are approaching each other on reciprocal or near-reciprocal courses. Both ships should alter their courses to starboard so that each shall pass on the port side of the other.

(2) Crossing: target ship approaching from A, B or D region in Fig. 1. The own ship and target ship are crossing each 
others' intended paths and so there is a risk of collision. The own ship is the stand-on ship and keeps its course and speed when the target ship is crossing from port to starboard of the home ship (D region in Fig. 1). If the target ship fails to take action, the home ship itself should substantially alter its course. The home ship is the give-way ship when the target ship is crossing from starboard to port of the home ship (A region in Fig. 1). If there is sufficient sea area, the own ship can alter its course substantially to starboard and cross from astern of the target. For the ship from $B$ region, if its relative angle with the own ship is great, a left turn can be taken to avoid collision.

(3) Overtaking: target ship approaching from region $\mathrm{C}$ in Fig. 1. A ship shall be deemed to be overtaking when another ship approaches from a direction more than 22.5 degrees abaft her beam. If a home ship is overtaking a target ship, the target ship is the no-deviation ship and should keep its course and speed. If the own ship is on the starboard quarter of the target ship, the own ship should alter its course to starboard. If the home ship is on the port quarter of the target ship, the home ship should alter its course to port.

\section{SHIP SAFETY DOMAIN RULE SETTING}

The concept of ship safety domain plays a very important role in ship collision avoidance and has been widely applied in marine traffic engineering, for risk assessment and VTS design. A series of related safety domain concepts have also been successively proposed. Goodwin [10] suggests a model based on the theory of 'ship domain'. A ship domain can be regarded as the sea around a ship that the navigator would like to keep free of other ships and fixed objects. Based on this concept, researchers have since proposed improvements to the ship domain model. Davis et al. [6] enhanced the model by adding the concept of an 'arena'. A ship arena is a larger domain based on the distance from another ship, at which a mariner would start to take action in order to avoid a close quarter situation. Colley et al. [5] proposed the Range to Domain and Range Rate model (RDRR). Each of these models endeavors to address the navigator's concern with respect to the physical separation of ships and their perception of possible ship-ship encounters when regions (domain or arena) become populated with other ships [23]. However, the ship safety domain, apart from being affected by the present encountered situation, would also vary according to different ship type, maneuverability and marine environment conditions. Hence, a unified safety domain or range cannot be set for all ships. In this regard, Zhu et al. [22] have considered the current visibility, ship maneuverability and closest point of approach (CPA) azimuth as the input factors, using neural networks to obtain projections based on different ship types and visibility situations in ship safety domains. This research is based on the fuzzy guard ring model by Kao et al. [14]. This model uses fuzzy logic as the method for model construction and factors include ship dimensions, ship speed and sea conditions as the model's input language variables. Through a fuzzy rule base and fuzzy inference, the fuzzy safety domain is calculated for the ship's guard ring. According to different situations, different radii are calculated for the fuzzy guard ring. Besides this, the navigator is also allowed to set the safety domain size according to the present situation. After the safety domain output value is obtained, the collision avoidance route planning model can then:

(a) Assess the risk of collision, select collision avoidance action according to the selected collision avoidance target.

(b) Confirm the time of latest collision avoidance turning.

(c) Confirm the steering angle necessary to safely control collision avoidance.

(d) Decide course return time.

(e) Confirm the steering angle necessary for safe course return.

\section{COLLISION AVOIDANCE PATH PLANNING PRINCIPLES}

This research uses the e-navigation concept to integrate the digital navigation information of each navigation equipment within the bridge, including own ship navigation course from the gyro compass, ship speed from the electronic log, ship position from the GPS and various weather information (for instance, wind direction, wind speed, current speed, current direction, etc). This information can then be used as input information for the ship safety domain calculation and basic ship settings for the collision avoidance model. As for other ship information necessary for the collision avoidance model, this is mainly real-time information from the target ship derived through AIS. The AIS data packet includes two major types of data, i.e., dynamic and static data. The AIS static data packet encompasses a ship's basic information, including ship name, destination, ship length, ship width, draft, tonnage, cargo type, ETA, etc., which is helpful towards understanding information about the target ship's basic characteristics. Within that information, ship length, width, draft and tonnage information further benefits an understanding of the target ship's maneuverability and safety domain calculation. AIS dynamic data consists of information about the target ship's real-time positional changes, which includes the ship's longitude and latitude positions, course, speed, etc.; serving as target ship input parameters for collision avoidance model calculations. AIS's reception range is approximately 20 nautical miles; when the target ship is within AIS's observation range, then according to the target ship's real time motion position obtained from AIS, we calculate the relative motion direction between own ship and the target ship, the distance of CPA (DCPA) and the time of CPA (TCPA). Furthermore, the knowledge base constructed by COLREGS can be used to decide the state of encounter, determining whether the ship is the give-way ship. If the ship is a give-way ship and it has a high risk of collision with the target ship, then this research's decision 
support system executes collision avoidance path planning to recommend a safe and economical path. According to the different task phases, the path solution can be divided into four phases:

(1) Cruising phase: for general navigational states, the system will remain in alert and search conditions at all times. It will also, based on the present navigation information and the calculated ship safety domain size through AIS information, set the alert range during collision avoidance (hence satisfying a situation where the ship wants to maintain a minimum distance from the target ship for safe passage).

(2) Initial warning phase:

(a) Collision avoidance alert encountered by single target ship: if a target ship enters the observation range, then the COLREGS knowledge base is used to determine the encounter state and collision risk. If it has been determined that the target has a collision risk (i.e. CPA less than the ship's safety domain alert range) and the ship is to give way, the system will proceed with collision avoidance route planning. In this phase, the system will determine, based on the original sail direction and speed, the amount of time required to arrive at the latest turning point. A greater steering angle might be required after passing this point. The navigator can use this information as the reference for the wheelbase turning point during collision avoidance. He/she can also simulate settings with different safe passage distances to obtain different turning time points, which can be used for turning point alerts.

(b) Collision avoidance alert encountered by multiple target ships: when there are many target ships within the observation range, the COLREGS knowledge base is first used to determine the encounter conditions and collision risks between the own ship and each target ship, determining which target ship is to make way for the own ship. Then, for each of these target ships, the DCPA between each target ship and the own ship is calculated. When the DCPA is less than the preset guarding ring values, it means that there is a collision risk between the target ship and the own ship. Then, among the target ships that meet this condition, the target ship that has the smallest TCPA is the one with the highest collision risk. Hence, this is the first ship in which the collision avoidance procedure for single target ship is executed. This collision avoidance procedure would also be assessed to determine whether it will impose a risk on other target ships. If it will, then it is corrected before being executed again. Following this principle, each multiship encounter is decomposed into individual encounter collision avoidance action cases between the home ship and each target ship [7].
(3) Collision avoidance navigation phase: for the path of collision avoidance turning, the turning angle should not be so small that the target ship is unable for to sense the intention of the home ship to avoid collisions, but it also should not be so large that it deviates too much from the original route. It should be ensured that it passes the target ship outside the safe alert range and that it only returns to course safely after a predefined period. An alert can be given prior to course return.

(4) Course return phase: when deciding the time and the course return operation strategy, it needs to be ensured that the course return operation will not result in new encounter danger states and that we minimize any unnecessary voyage time loss due to further collision avoidance actions. The returning point, and returning time alerts, can also be provided prior to restore to the original route.

\section{SHIP COLLISION AVOIDANCE ROUTE PLANNING BY ANT COLONY ALGORITHM}

\section{Description of Ant Colony Algorithm}

Research has shown that ant colonies naturally possess intelligence and are able to find the shortest route from a food source to their nests without any visual aids, but through the use of pheromones emitted by the ants as cues. The ant colony algorithm is a form of evolutionary computation that models the behavior of real ants in search of food. This algorithm was proposed by Dorigo [8] and has been successfully used to solve numerous real-life problems, such as the traveling salesman problem (TSP). As a whole, the ant colony algorithm has a unified framework model. It is robust, embodies positive feedback and distributed computing characteristics. Hence, the ant colony algorithm is well suited to collision avoidance. This research utilizes the ant colony algorithm to enable collision avoidance for ships moving towards their goals. In short, this is achieved by choosing the most suitable objective function during the collision avoidance route search so that the search process becomes more efficient and effective.

As for the application of evolution computation to route planning, it has already been used for obstacle avoidance in robots [16], and the same principles could be applied to ship collision avoidance. Smierzchalski and Michalewicz [19] treated the longitude and latitude of turning points on collision avoidance routes as the genetic code, and added ship speed and time concepts to form the genes in the chromosomes, in order to search the optimum route. These two research studies address the route planning of robots mainly, so COLREGS was not included. One application of the ant colony algorithm is worked out by He and Qi [11], who have applied the algorithm to automatic underwater vehicle (AUV) collision avoidance. This research is similar to the traveling salesman method in the sense that the midpoints of routes are identified and the shortest route is determined by a number passing through the midpoints while satisfying collision avoidance requirements. 
However, the use of traditional ant colony algorithms for collision avoidance problems has mainly focused on directed graphs or grids, whereby the required search space is very large, making the search inefficient and thus, not suitable to be used directly for collision avoidance in ships. Furthermore, if grid format data were used to represent ships' navigational routes, too many turning points would be generated, and this does not meet ship navigational practice. On the contrary, this research uses vector format data as the basis of estimation for collision avoidance routes. This research employs the whole collision avoidance route as the basis of assessment, considers both safety and economy at the same time, and includes the factors of the COLREGS and safety domain, in order to inform collision avoidance measures, such as earliest turning point time, safe angle of collision avoidance, navigational restore time and navigational restore angle, etc.

\section{The Coding form of Collision Avoidance Routes}

In order to accelerate the search speed, instead of using the longitude and latitude of the turning points as the nodes of the directed graph, this research uses the following four parameters of collision avoidance routes for encoding:

(a) The required time to the turning point (or the time from TCPA) $T s$. This parameter represents the latest turning point. Collision avoidance turning needs to occur before this time, else there is a collision risk.

(b) The required collision avoidance angle for passing the target ship at safe distance $C_{O}^{\prime}$. The collision avoidance angle needs to be at least larger than $C^{\prime}{ }_{O}$, else there exists a collision risk.

(c) The time between the turning to collision avoidance and the turning to navigational restore $T a$. After navigating at angle $C^{\prime}{ }_{O}$ to avoid collision, the avoidance course must be run for at least $\mathrm{Ta}$ minutes before a return to course can be considered.

(d) The limited angle upon turning of navigational restore $C b$. After navigating at angle $C^{\prime}$ for at least $T a$ minutes, if there is a return to course, the navigation angle must be limited to below $C b$ value, or a near collision state will still result.

These four parameters can constitute a collision avoidance route as a series of nodes in the directed graph and also form important parameters for collision avoidance alerts. Considering the ship speed and observation distance, we assume that the ship can arrive in 100 minutes. It can be noted that two digits can fully express the values within 100 . When the relative bearing of the bow is 'head up', the safe collision avoidance angle $C^{\prime}{ }_{O}$ should be limited to within $30 \sim 90$ degrees at the starboard side, in order to prevent over turning or insufficient turning (which is also expressed by two digits). The time between the turning to collision avoidance and the turning to navigational restore $T a$ should not exceed 60 minutes. When

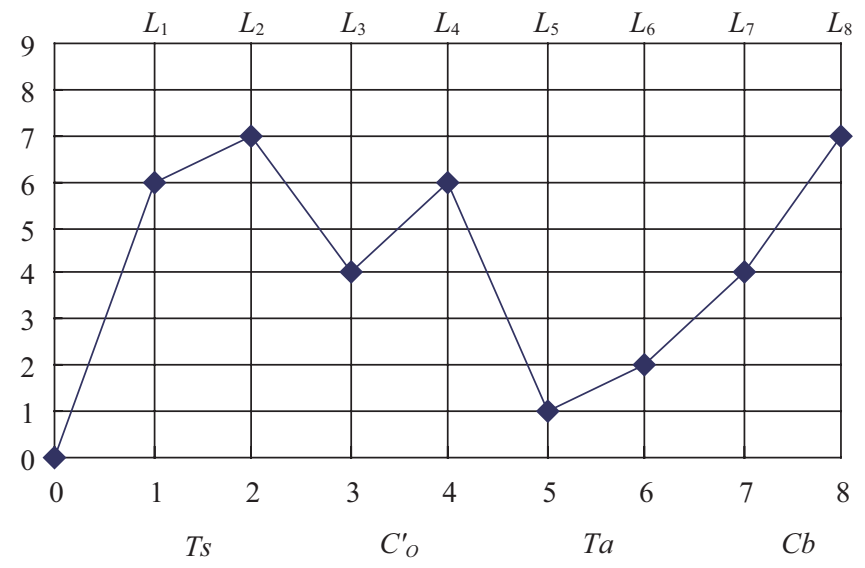

Fig. 2. Nodes and route planning illustration.

the relative bearing of bow is head up, the turning angle of navigational restore $C b$ should be limited to within $30 \sim 90$ degrees at port side. To use these parameters for the ant colony algorithm, the four parameters should be represented in the $\mathrm{X}-\mathrm{Y}$ axis, as shown in Fig. 2. Eight even vertical lines are then drawn on the graph across the graph and labeled as $L_{1}$, $L_{2}, \ldots, L_{8}$, with $L_{1} \sim L_{2}, L_{3} \sim L_{4}, L_{5} \sim L_{6}$ and $L_{7} \sim L_{8}$ representing $T s, C_{O}^{\prime}, T a$ and $C b$ respectively. These line segments are represented numerically from 1 to 8 in the figure shown. Every line segment has 9 equal parts and as such, each line segment has 10 nodes, representing the value of each line segment ranging from 0 to 9 . The graph has a total of $8 \times 10$ nodes, and every node can be represented by $\operatorname{Knot}\left(x_{i}, y_{i, j}\right)$, where $x_{i}$ represents the x-coordinate for $L_{i}(i=1 \sim 8), y_{i, j}$ represents the y-coordinate for node $j$ of line segment $L_{i}(j=0 \sim 9)$. Every node represents the y-coordinate value $y_{i, j}$. For instance, $\operatorname{Knot}(7,2)$ represents a value of 2 for the first digit of $C b$.

Assume that an ant starts moving from origin $O$. When it climbs to line segment $L_{8}$ and finishes one cycle, its climb can be represented as: Path $=\{O, \operatorname{Knot}(x 1, y 1, j), \operatorname{Knot}(x 2, y 2$, $j), \ldots, \operatorname{Knot}(x 8, y 8, j)\}$. Here, node $\operatorname{Knot}(x 2, y 2, j)$ is situated at line segment $L_{i}$ and thus, the parameters $T s, C^{\prime}{ }_{O}, T a$ and $C b$ for this climb can be calculated as follows:

$$
\left\{\begin{array}{l}
T s=y_{1, j} \times 10+y_{2, j} \\
C^{\prime}{ }_{O}=y_{3, j} \times 10+y_{4, j} \\
T a=y_{5, j} \times 10+y_{6, j} \\
C b=y_{7, j} \times 10+y_{8, j}
\end{array}\right.
$$

Figure 2 illustrates the climb route for an ant. This route represents the collision avoidance route parameter values as: $T s=67, C_{O}^{\prime}=46, T a=12, C b=47$.

The four parameters proposed efficiently represent the climb route for the ant and consequently improve the efficiency of calculations for the algorithm. Furthermore, the optimal combination of parameters is desirable for sea navigation because 
it is not only a set of turning point values and connection distances but would provide useful references, upon further analysis and interpretation.

\section{Objective Function}

The technique of selecting a rational and effective collision avoidance measure depending on the state of encounter between the home ship and a target ship is the focus of this research. We will use the same objective function as those used by Tsou et al. [21]. In the development of this technique, this research maintains a safe domain distance between the home ship and the target ship, articulates the turning angle and time, the navigational restore time and the navigational restore angle. It also prevents the generation of new encounter states or domain stress phenomena. The distance from the beginning of the turning to the restoring of the original route is the objective function between the home ship and the target ship. The ACA is used to obtain the shortest collision avoidance route for meeting the objective function and constraint conditions, so that the home ship can satisfy the following:

(a) The total distance of collision avoidance will be minimal.

(b) The risk of collision will be minimal and the target ship will be kept outside the safe domain in avoidance.

(c) Under the safety domain passing conditions, collision avoidance angles shall be minimized.

(d) After the least time of navigational detour, the home ship resumes its original route.

(e) Where there are no new encounters or other domain stress phenomena, the turning angle shall be minimized.

Assume that the course $C_{T}$, speed $V_{T}$, bearing $Q$, distance $D$ of the target ship, and the course $C_{O}$, speed $V_{O}$ of the home ship are known. If $C^{\prime}{ }_{O}$ is the new course of the home ship after collision avoidance, then the fitness function is:

$$
\text { Distance }=\min _{i=1}^{n}\left\{D s_{i}+D r_{i}\right\}
$$

where $D s_{i}$ is the distance after collision avoidance, $D r_{i}$ is the distance of navigational restore.

$$
D s_{i}=\text { ColAvTime } * V_{O}, D r_{i}=\text { ResTime } * V_{O}
$$

ColAvCourse is the turning angle after collision avoidance.

ResCourse is the turning angle of navigational restore.

ColAvTime is the navigation time after collision avoidance. ResTime is the navigation time of navigational restore.

The constraint conditions are:

$$
\begin{gathered}
30 \leq \text { ColAvCourse } \leq 90 \\
-60 \leq \text { ResCourse } \leq-30 \\
T C P A_{1} \leq \text { ColAvTime } \leq 60
\end{gathered}
$$

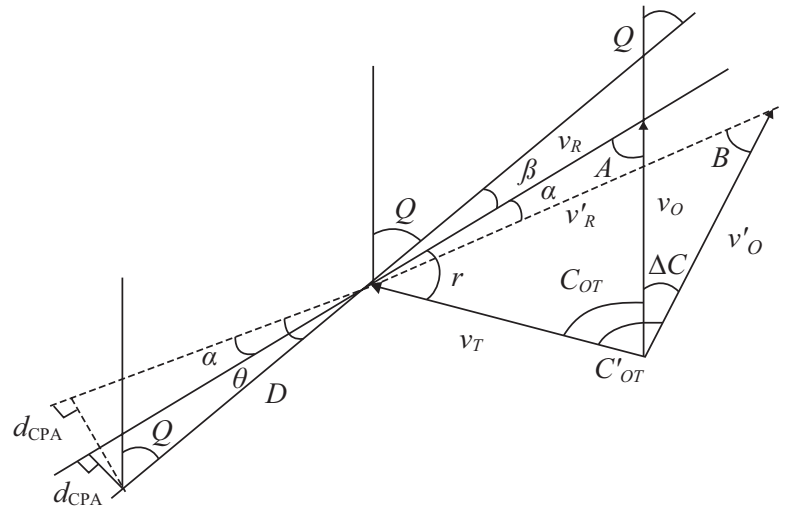

Fig. 3. Collision avoidance decision model after the home ship is turned [17].

$$
D C P A_{1} \geq G d \text { and } D C P A_{2} \geq G d
$$

where ColAvCourse $\in[30,90]$ is the decision parameter, which represents the turning angle of collision avoidance (the difference of angle between the new course and the original course, and a positive value means right turn), ResCours $\in$ $[-60,-30]$ represents the turning angle of navigational restore (the difference of angle between the new course and the original course, and a negative value means left turn). The navigational restore time should not exceed 60 minutes and should be at least larger than $T C P A_{1}$ time (new TCPA time after collision avoidance). $D C P A_{1}$ and $D C P A_{2}$ are the new DCPA after collision avoidance and the new DCPA of navigational restore, respectively.

Combining the above mentioned requirements, it can be observed that obtaining DCPA and TCPA is an important factor for satisfying objective function constraint requirements. We reference Hollingdale [12] and Liu et al. [17] for the calculation method for DCPA and TCPA, and further modify it to make it more suitable for calculation in programming languages. As shown in Fig. 3, if the turning angle of the home ship is ColAvCourse (right + , left -), and the new angle between the home ship and the target ship is $C^{\prime}{ }_{O T}$ (range: $-180^{\circ} \sim$ $180^{\circ}$ ), then new $D C P A_{1}$ and $T C P A_{1}$ will be calculated as follows:

$$
\begin{gathered}
D C P A_{1}=D \sin \theta \\
T C P A_{1}=D \cos \theta / V_{R}^{\prime}
\end{gathered}
$$

where $\theta$ is the angle between the relative motion line of the home ship and the bearing of the target ship; $D$ is the distance between home ship and the target ship; $V_{R}^{\prime}$ is the relative speed after collision avoidance; and $C_{O T}^{\prime}$ is the angle between the home ship and the target ship after the home ship is turned.

$$
C_{O T}^{\prime}=C_{O}^{\prime}-C_{T}
$$


If $C_{\text {OT }}^{\prime} \geqq 0^{\circ}$, then $\theta=B+$ ColAvCourse $-Q$

If $C_{O T}^{\prime}<0^{\circ}$, then $\theta=-(B+$ ColAvCourse $-Q)$

$V_{R}^{\prime}$ is the relative speed after the home ship is turn:

$$
V_{R}^{\prime}=\sqrt{V_{T}^{2}+V_{O}^{2}-2 V_{T} V_{O} \cos C_{O T}^{\prime}}
$$

If $B$ is the angle between the relative motion line of the home ship and the head line after the home ship is turned, then the way of calculation is as follows:

$$
\text { If } C_{O T}^{\prime} \geqq 0 \text {, then }
$$

$$
B=\cos ^{-1}\left(\left(V_{O}^{2}+V_{R}^{\prime 2}-V_{T}^{2}\right) / 2 V_{O} V_{R}^{\prime}\right)
$$

If $C^{\prime}{ }_{O T}<0$, then

$$
B=-\cos ^{-1}\left(\left(V_{O}^{2}+V_{R}^{\prime 2}-V_{T}^{2}\right) / 2 V_{O} V_{R}^{\prime}\right)
$$

$G d$ represents the radius of the guarding ring for safe passing, which means that the new DCPA should be at least larger than the radius of the guarding ring. This value depends on marine conditions and ship type, which can be obtained from self setup or other models.

\section{Route Selection}

Assume that each time it takes for every ant to climb from a node in line segment $L_{i}$ to another node in line segment $L_{i+1}$, regardless of the distance between the nodes. If all the ants embark from the origin coordinate $O$, then they will arrive at every line segment $L_{i}(i=1 \sim 8)$ at the same time and finally, arrive at their individual final node at $L_{8}$, finishing one cycle.

At time $t$, assume that the ant colony moves to line segment $L$. Then, $b_{j}(j=0 \sim 9)$ represents the number of ants at node $j$ on $L_{i}$. This number of ants can be represented by $m$, where $m=\sum_{j=0}^{9} b_{j}(t)$. Let $\tau\left(x_{i}, y_{i, j}, t\right)$ be the remaining information at node $\operatorname{knot}\left(x_{i}, y_{i, j}\right)$ at time $t$. At the start time, all nodes have the same quantity of information, i.e. $\tau\left(x_{i}, y_{i, j}, 0\right)=c$ (where $c$ is the quantity, $i=1 \sim 8, j=0 \sim 9), \Delta \tau\left(x_{i}, y_{i, j}, 0\right)=c$. Let $P_{k}\left(x_{i}, y_{i, j}, t\right)$ be the probability of climb for ant $k$ from a node on $L_{i-1}$ to $\operatorname{knot}\left(x_{i}, y_{i, j}\right)$ at time $t$, where

$$
P_{k}\left(x_{i}, y_{i, j}, t\right)=\frac{\tau^{\alpha}\left(x_{i}, y_{i, j}, t\right) \eta^{\beta}\left(x_{i}, y_{i, j}, t\right)}{\sum_{j=0}^{9} \tau^{\alpha}\left(x_{i}, y_{i, j}, t\right) \eta^{\beta}\left(x_{i}, y_{i, j}, t\right)}
$$

and $\eta\left(x_{i}, y_{i, j}, t\right)$ as the visibility of $\operatorname{knot}\left(x_{i}, y_{i, j}, j\right)$ represented by

$$
\eta\left(x_{u}, y_{i, j}, t\right)=\frac{10-\left|y_{i, j}-y_{i, j}^{*}\right|}{10}
$$

In (12), the value of $y^{*}{ }_{i, j}(i=1 \sim 8, j=0 \sim 9)$ can be obtained as follows: At the first cycle, $y_{i, j}^{*}$ is the random value that satisfies the set constraints, corresponding to the coordinate values of the 8 nodes in Fig. 2. At the later cycles, $y_{i, j}^{*}$ corresponds to the coordinate values of the 8 nodes mapped through the parameter set $T s, C^{\prime}{ }_{O}, T a$ and $C b$ for the optimal route produced in the previous cycle.

\section{Information Update}

Assume that at the origin time $t=0$, all ants are positioned at the origin $O$. After 8 time units, all ants climb to the end point. The following functions can then be used to adjust the information quantity for various nodes:

$$
\begin{gathered}
\tau\left(x_{i}, y_{i, j}, t+8\right)=\rho \tau\left(x_{i}, y_{i}, t\right)+\Delta \tau\left(x_{i}, y_{i, j}\right) \\
\Delta \tau\left(x_{i}, y_{i, j}\right)=\sum_{k=1}^{m} \Delta \tau_{k}\left(x_{i}, y_{i, j}\right) \\
\Delta \tau\left(x_{i}, y_{i, j}\right)=\frac{Q}{F_{k}}
\end{gathered}
$$

In (15), $Q$ represents the information strength, which affects the convergence rate of the functions. $F_{k}$ represents the objective function value for the $\mathrm{k}^{\text {th }}$ ant in that cycle, which can be calculated via formula (1).

\section{Algorithm Steps}

The steps for using the ant colony algorithm to determine the parameter set for the shortest collision avoidance route are listed as follows:

(1) Random values that satisfy set constraints are used to calculate the start values for $T s, C^{\prime}{ }_{O}, T a$ and $C b$.

(2) Let the number of ants be $m$ and for each ant $k(k=1 \sim m)$, an 8 element sequence representing one route Path $_{k}$. Within $\mathrm{Path}_{k}$, we store the coordinate values for the 8 nodes that the $k^{\text {th }}$ ant will pass through, which can be used to represent the climb route of the $k^{\text {th }}$ ant or one collision avoidance route.

(3) The time counter, $t$ is then set as 0 , cycle number $N_{C}=0$, the maximum number of cycles set as $N_{C M A X}$, the information quantity of each node, $\left(x_{i}, y_{i, j}, 0\right)$, initialized as $\Delta \tau\left(x_{i}, y_{i, j}\right)=0(i=1 \sim 8, j=0 \sim 9)$ and each ant to start at origin $O$.

(4) Variable $i$ is set as 1.

(5) Formula (11) is used to calculate the transfer probability at each node for the ant approaching line segment $L_{i}$. Ac- 
cording to the calculated probability, a node is chosen for each ant $k(k=1 \sim m)$ at $L_{i}$ in the roulette manner. The ant $k$ is then transferred to that node chosen and the coordinate value of the node is stored in the $i^{\text {th }}$ element of $P a t h_{k}$.

(6) Set $i=i+1$. If $i<=8$, return to Step (5), else continue to Step (7).

(7) According to the route taken by the $\mathrm{k}^{\text {th }}(k=1 \sim m)$ ant, i.e. Path $_{k}$, formula (1) can then be used to calculate the corresponding parameters $T s^{k}, C^{\prime}{ }^{*}{ }^{*}, T a^{k}$ and $C b^{k}$ for the route. Following this, formula (2) can be used to calculate the objective function value $F_{k}$ for ant $k$. The best route for the current cycle (corresponding to the best parameter set for the current cycle) would then be recorded and the respective parameter sets in $T s^{*}, C^{\prime}{ }_{O}^{*}, T a^{*}$ and $C b^{*}$.

(8) Set $t \leftarrow t+8, N c \leftarrow N c+1$. According to formula 13, 14, 15 , the information quantity at each node is renewed and all elements in $\operatorname{Path}_{k}(k=1 \sim m)$ are reset to zero.

(9) If $N c<N c m a x$ and the ant colony has not converged to following the same route, the positions of all ants are then reset to $O$ and we revert to Step (4). If $N c<N c \max$ but the ant colony has converged to the same route, the calculation is complete and the best route and corresponding parameter set combinations are obtained.

\section{EXPERIMENT RESULTS}

\section{E-Navigation Platform Deployment}

Under the e-navigation concept, the Electronic Chart Display and Information System (ECDIS) plays a very important role. It can be described as a platform for aggregation, analysis, display and operation of various kinds of information, serving as the interface for human-computer communication. There are many ways to realize ECDIS but they are mostly related to GIS. The system can be directly implemented on the GIS platform or by embedding GIS's COM component into a general information system to achieve ECDIS functionality. As the collision avoidance model involves spatial attributes and geometric computation, if we make use of the spatial analysis and geometric functionality provided by GIS, computational efficiency and model results will be greatly improved. This research did not deploy the system based on existing GIS software but instead, used Visual Basic. Net as the program development tool, while ESRI's MapObject COM component provided the platform for GIS functionality, seamlessly embedding spatial analysis and display functionality of the GIS components into a general information system. This is combined with each module in this research, customizing it to provide the spatial decision support system that is required for collision avoidance route planning. This method makes execution more efficient and program I/O interface more open, facilitating real-time exchange and the integration of information with other navigation instruments on the bridge/ navigation information systems [3], constructing an Integrated Bridge System (IBS) which satisfies the e-navigation objec-

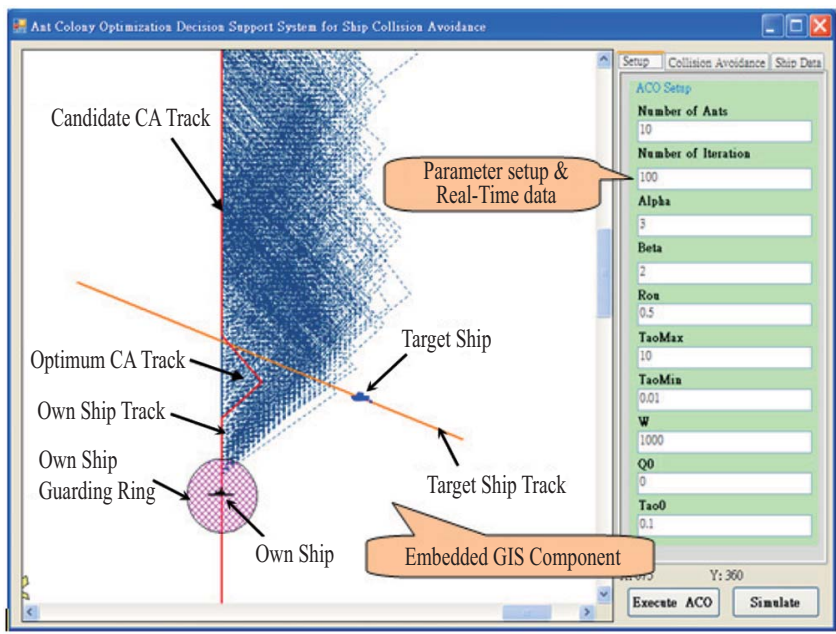

Fig. 4. Ant colony algorithm path planning for collision avoidance on a e-navigation platform.

tive. Figure 4 shows the system's user interface, the left half showing the GIS component's collision avoidance path spatial control and display, consisting of the home ship's location, alert range and path, the target ship's position and path, candidates collision avoidance paths generated by the ant colony algorithm (dotted line regions) and the final optimum collision avoidance path obtained. The right half shows the related ant colony algorithm, parameter settings for collision avoidance principles as well as target ship and home ships' real-time data and DCPA, TCPA solutions. Through the ant colony algorithm, we can simulate collision avoidance measures in the situations as well as displaying the whole collision avoidance process one by one in real-time simulation, allowing us to visually assess the collision avoidance route.

\section{Simulation Results}

As below, using the settings in Fig. 4, we discuss the simulation results for single ship and multiple ships encounters and the related ant colony algorithm parameter values. These values reference Duan's [9] recommended execution setting, where the number of ants is set as $10, \alpha=3, \beta=2, \rho=0.5, Q=1000$ and 100 iterations being executed. Movement information comes from AIS, the target ship's sail direction is fixed, with movement speed set at 15 knots. Home ship speed is set at 14 knots, angle at 000 , changing course only to carry out collision avoidance path planning.

\section{1) A Single Ship Encounter Situation}

Here, we separately simulate three types of encounter states during avoidance, based on the collision avoidance principles. According to the ant colony algorithm, there are three types of avoidance course with the shortest collision avoidance path: Case 1-intersection encounter coming from the upper right (Fig. 5), shown as region A in Fig. 1. Case 2: intersection encounter coming from lower right (Fig. 6), shown as region B in Fig. 1. Case 3: head-on encounter (Fig. 7), shown as region 


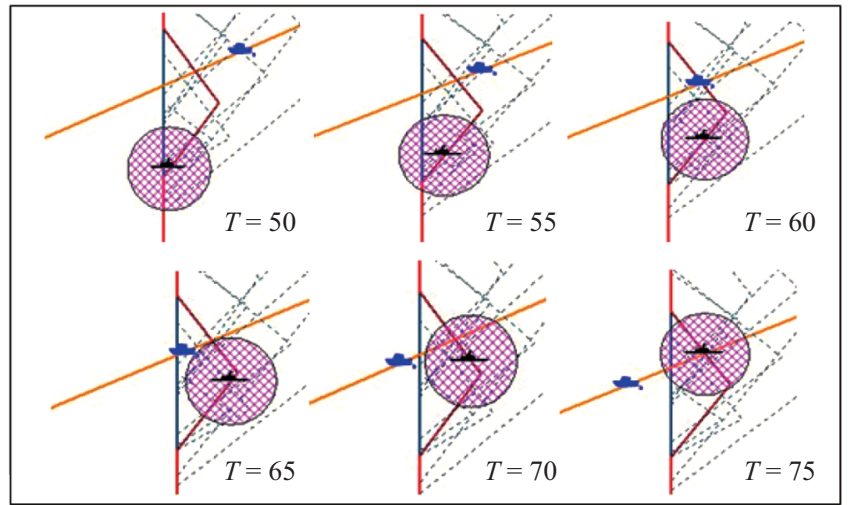

Fig. 5. Intersection encounter from upper right direction, dynamic simulation of collision avoidance path.

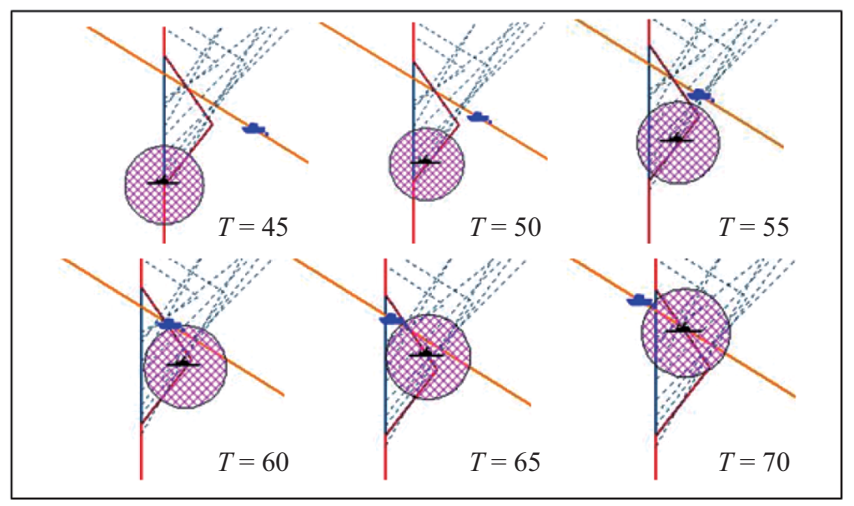

Fig. 6. Intersection encounter from lower right direction, dynamic simulation of collision avoidance path.

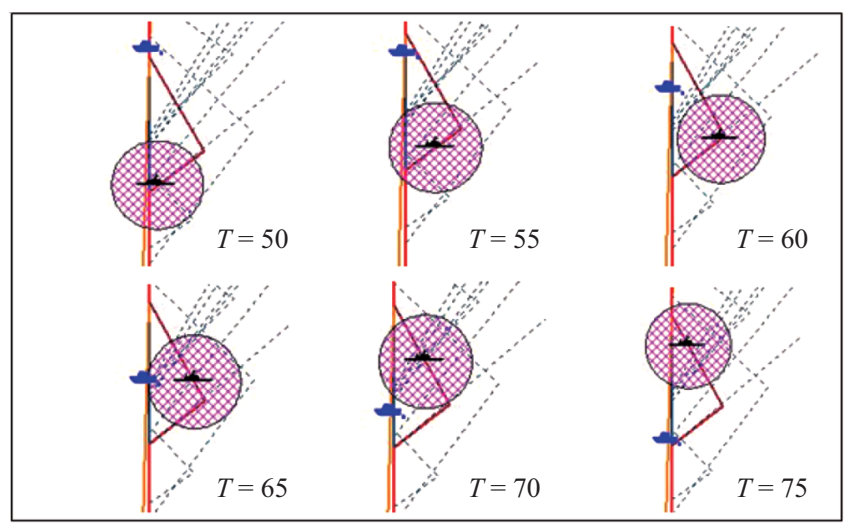

Fig. 7. Head-on encounter, dynamic simulation of collision avoidance path.

E in Fig. 1. The time values shown in Figs. 5, 6 and 7 represent the time passed (in minutes) since start of observations. After simulation verification, actual setting requirements can be observed, satisfying navigational practices for safe passage. Figure 9 uses case 1 as an example, separately using the ant colony algorithm and the widely popular evolutionary algorithm, Genetic Algorithm (GA) used by Tsou et al. [21], to

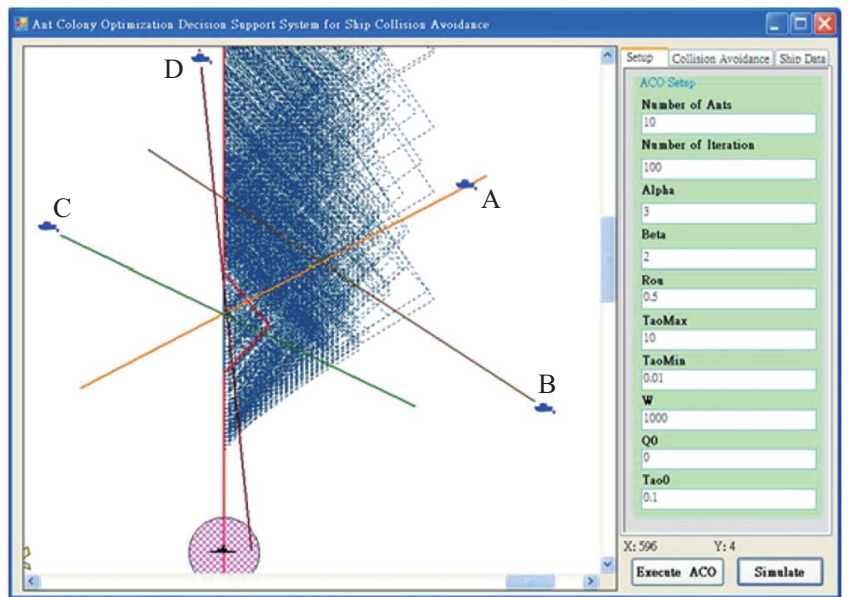

Fig. 8. Situations to determine and collision avoidance action for multi ship encounters.

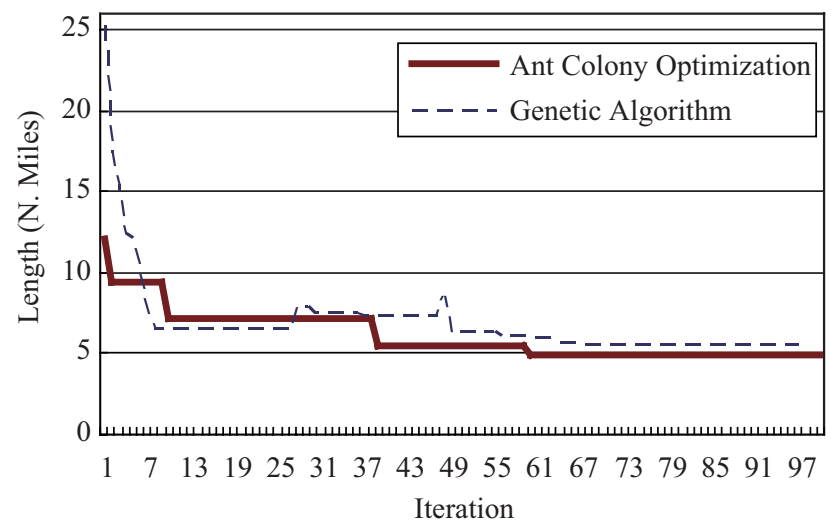

Fig. 9. ACA and GA execution results.

conduct 100 computation iterations for actual comparison, showing the change in collision avoidance path length during the evolutionary convergence process. In these comparison experiments, GA also uses the same 4 parameters as the genetic code, executing related genetic operations such as reproduction, crossover and mutation. After observing several experimental results, we note that although the genetic algorithm rapidly converges and can generate sudden genetic change (like No. 28, No. 46 cycles) to exceed the local optimum, the ACA can stably converge and the final result obtained is not inferior to the result from GA computation. Furthermore, the collision avoidance path obtained through ACA not only maintains safe encounter but also is the most economical path that satisfies constraint conditions. Table 1 presents the simulation values and the ant colony algorithm path planning values for the three cases. In the table, the DCPA and TCPA represents the CPA distance and time between the own ship and target ship in the initial state when no collision avoidance measures have been taken. When DCPA is positive, it means that the target ship has passed from the home ship's front. When DCPA is negative, it means that the target ship 
Table 1. The recommended data for simulation setup and collision avoidance actions of ant colony algorithm.

\begin{tabular}{|c|c|c|c|c|c|c|c|c|c|c|}
\hline \multirow{2}{*}{ Case } & \multirow{2}{*}{$\begin{array}{c}\text { Target Ship } \\
\text { Course }\end{array}$} & \multirow{2}{*}{$D C P A$} & \multirow{2}{*}{ TCPA } & \multicolumn{4}{|c|}{ CA Measures } & \multirow{2}{*}{$\begin{array}{c}\text { Initial } \\
\text { Bearing }\end{array}$} & \multirow{2}{*}{$\begin{array}{c}\text { Iinitial } \\
\text { Distance }\end{array}$} & \multirow{2}{*}{ Length } \\
\hline & & & & $T 1$ & Co 1 & $T 2$ & Co 2 & & & \\
\hline 1 & 246 & 0.74 & 73 & 67 & 47 & 13 & -46 & 41 & 28 & 5.85 \\
\hline 2 & 287 & -0.23 & 81 & 61 & 53 & 15 & -55 & 79 & 23 & 6.78 \\
\hline 3 & 191 & 0.43 & 67 & 52 & 44 & 13 & -37 & 2 & 33 & 6.32 \\
\hline
\end{tabular}

has passed from the home ship's rear. The effect achieved from executing ACA is similar to the result obtained by GA and the collision avoidance measure follows the recommended path as derived from the result of ACA. $T 1$ represents the time in minutes after which the ship must start to turn to avoid a collision (else a larger turning degree is needed), so that the target ship can pass outside the alert range. $C 1$ is the collision avoidance turning angle (right turn), limited to between 30 degrees and 60 degrees to the right of the intersection line. $T 2$ represents the time in minutes after collision avoidance when the ship can return to course. It is the shortest time required for collision avoidance to ensure safe passage, whereby if a return to course is done in less than this time, there will be a risk of collision. $C 2$ is the return to course turning angle (intersection angle with original path), limited to between 30 degrees and 60 degrees to the left of the intersection line. If the angle taken is larger than $C 2$, then there is a risk of approaching a collision. In order to allow safe encounters between ships, regardless of whether it is the collision avoidance or return to course phase, the DCPA between other ships should remain outside the alert range. Using Fig. 7 as an example, when the home ship is at $T=60$, the collision avoidance measure taken just allows safe passage and when $T=65$ and $T=70$ is taken for return to course, though the ship is near the target ship, it still remains outside the alert range of the target ship.

\section{2) Multiple Target Ships Encounter Situations}

In principle, we base the collision risk assessment on the collision avoidance knowledge base and divide the multiple target ships to be assessed as single encounters one after the other with the own ship. Using Fig. 8 as an example, in this multiple ship encounter situation, there are four ships A, B, C, $\mathrm{D}$, which are the target ships that will be encountered by the own ship. Here, A is the upper right target ship that is crossing the own ship with $D C P A=0.52, T C P A=49, \mathrm{~B}$ is the lower right target ship that is crossing own ship with $D C P A=0.42$, $T C P A=73, \mathrm{C}$ is the left target ship that is crossing own ship with $D C P A=0.34, T C P A=68$ and $\mathrm{D}$ is the head-on target ship that is crossing own ship with $D C P A=0.24, T C P A=64$. From decisions made from the knowledge base, the DCPAs of the four target ships are all less than the preset 2 nautical miles of the alert range, exceeding the threshold collision risk and thus, a risk of collision. As $\mathrm{C}$ is the ship that gives way, it is first to be removed. Next, we target ships A, B and D. Although the DCPAs of ships B and D are less than that of A, according to the knowledge base decisions, ship A has the smallest TCPA and hence, will be the earliest to collide. Hence, the system will give priority to ship A to execute collision avoidance path planning and, after it is completed, the situation is reassessed and collision avoidance path planning is continued.

\section{3) Execution Efficiency}

Regarding execution time efficiency, though the overall program has not reached the optimal algorithmic efficiency, we use XP Professional Edition as the environment to conduct the PC simulation, averaging an execution time between 10 and 20 seconds. Under the same conditions, the GA takes between 14 and 26 seconds. The ant colony algorithm already satisfies navigation decision requirements and if its inherent parallel processing capability can be strengthened, real-time decision support can be provided.

\section{CONCLUSION}

Following the increased adoption of new navigation instruments, present navigators lack not so much sufficient information, as the capability to rapidly and effectively assimilate and use the navigation information to make the most correct decision. Therefore, through using appropriate decision support tools to aid in the handling of navigational decision making, the number of mistakes due to subjective human judgments can be reduced and sea traffic safety improved. As this is the main perspective that e-navigation advocates, this research used the concept of e-navigation as its framework. It combines related navigation information, targets the most common problems encountered during navigation in collision avoidance decision support and applies the ant colony algorithm in the field of artificial intelligence. This is to construct a life-form optimization behavior-based collision avoidance model. This model integrates navigation practices, a maritime regulations knowledge base and navigational information from AIS, as well as using GIS as ECDIS information display and a platform for execution. Through our experiments, it has been shown that the ant colony algorithm outperforms the mainstream evolutionary computation method (e.g. GA) with respect to both execution efficiency and execution results. This enables planning of suitable navigation collision avoidance strategy recommendations, useful for reducing navigator workload. The path planned, simultaneously considers both economy and safety, while being the safety critical, shortest collision avoidance route. Through this planning, the latest avoidance time can be recommended, with the minimal turning degree, the 
earliest course return after avoidance and the largest course return degree. Although in the practice of decision making, the navigator may not use such an avoidance path, this path nevertheless represents a safe and short passage, while the turning angle and times can serve as the reference for safe threshold values or alert. This reference information is not only meaningful to the navigator but also provides VTS operators with a reference for an assessment of territorial waters traffic. Although at present this research has only been applied for collision avoidance short distance navigation, the theory can be applied in the same way in the future to long distance navigation planning. Apart from this, we can note that in the setting of ship safety, this research applied the design of a circular guard ring. In the future, other shapes for ship domain models can be considered, in order to design a collision avoidance alert region that better satisfies the present situation. With the growing popularity of the distributed environment, there has been widespread application of a parallel or distributed processing nature. Therefore, in consideration of the benefits of distributed processing, the collective intelligence feedback mechanism characteristics of the ant colony can be manipulated on the parallel processing environment. Through this distributed and parallel processing infrastructure, the ant colony algorithm computational performance can be made more efficient and given the efficiency to surpass other solutions. This will also better satisfy real-time decision support requirements in navigation.

\section{ACKNOWLEDGMENTS}

It is appreciated that this research is subsidized by funding from the National Taiwan Ocean University (NTOU-RD97201-03-34-01).

\section{REFERENCES}

1. Alexander, L., "E-navigation: concept vs. reality," Sea Technology, Vol. 4, No. 1, pp. 12-15 (2009).

2. Bao, J.-B., "The development of the concept of e-navigation (In Chinese)," China Maritime Safety, Issue 11, pp. 48-51 (2007).

3. Beaubouef, T. and Breckenridge, J., "Real-world issues and applications for real-time geographic information systems (RT-GIS)," The Journal of Navigation, Vol. 53, No. 1, pp. 124-131 (2000).

4. Cockroft, A. N., "Collision at sea," Safety at Sea, pp. 17-19 (1984).

5. Colley, B. A., "A marine traffic flow and collision avoidance computer simulation," The Journal of Navigation, Vol. 37, No. 2, pp. 232-250 (1984).

6. Davis, P. V., Dove, M. J., and Stockel, C., "A computer simulation of marine traffic using domains and arenas," The Journal of Navigation, Vol. 33, No. 2, pp. 215-222 (1980).

7. Davis, P. V., Dove, M. J., and Stockel, C. T., "A computer simulation of multi-ship encounters," The Journal of Navigation, Vol. 35, No. 2, pp. 347 352 (1982).

8. Dorigo, M., Manizzzo, V., and Colomi, A., "Ant system optimization by a colony of cooperating agents," IEEE Transaction on System, Man and Cybernetics- Part B: Cybernetics, Vol. 26, No. 1, pp. 29-41 (1991).

9. Duan, H.-B., Ant Colony Algorithm: Theory and Application, Science Press: Peking, China, pp. 100-101 (2005).

10. Goodwin, E. M., "A statistical study of ship domains," The Journal of Navigation, Vol. 28, No. 3, pp. 328-344 (1975).

11. He, Z. and Qi, L., "Research of a method for AUV to avoid collision with moving obstacles based on Ant Colony Algorithm," (in Chinese), Ship Science and Technology, Vol. 29, No. 6, pp. 86-89 (2007).

12. Hollingdale, S. H., "The mathematics of collision avoidance in two dimensions," The Journal of Navigation, Vol. 14, No. 3, pp. 243-261 (1961).

13. Jones, K. D., "Decision making when using collision avoidance system," The Journal of Navigation, Vol. 31, No. 2, pp. 173-180 (1978).

14. Kao, S.-L., Lee, K.-T., Chang, K.-Y., and Ko, M.-D., "A fuzzy logic method for collision avoidance in vessel traffic service," The Journal of Navigation, 60, pp. 17-31 (2007).

15. Li, L.-N., Yang, S.-H., Cao, B.-G., and Li, Z.-F., “A summary of studies on the automation of ship collision avoidance intelligence," (in Chinese) Journal of Jimei University, China, Vol. 11, No. 2, pp. 188-192 (2006).

16. Lin, H. S., Xiao, J., and Michalewicz, Z., "Evolutionary algorithm for path planning in mobile robot environment," IEEE World Congress on Computational Intelligence., Proceedings of the First IEEE Conference, pp. 211-216 (1994).

17. Liu, D., Wu, Z., and Jia, C., "Decision making model of DCPA, TCPA and object's movement parameter,'(in Chinese), Journal of Dalian Maritime University, China, Vol. 30, No. 1, pp. 22-25 (2004).

18. Notatet, E. S., "Marine eNavigation," http://www.itsnorge.no/maritime/ MARINEeNAVIGATION.html (2005).

19. Smierzchalski, R. and Michalewicz, Z., "Modeling of ship trajectory in collision situations by an evolutionary algorithm," IEEE Transactions on Evolutionary Computation, Vol. 4, pp. 227-241 (2000).

20. Statheros, T., Howells, G., and McDonald-Maier K., "Autonomous ship collision avoidance navigation concepts, technologies and techniques," The Journal of Navigation, Vol. 61, pp. 129-142 (2008).

21. Tsou, M. C., Kao, S. L., and Su, C. M., "Decision support from genetic algorithm for ship collision avoidance route planning and alerts," The Journal of Navigation, Vol. 63, No. 1, pp. 1-16 (2010).

22. Zhu, X., Xu, H., and Lin, J., "Domain and its model based on neural networks," The Journal of Navigation, Vol. 54, No. 1, pp. 97-103 (2001).

23. Zhuo, Y. and Hearn, G. E., "A ship based intelligent anti-collision decision-making support system utilizing trial manoeuvres," Control and Decision Conference, CCDC 2008, Chinese, pp. 3982-3987 (2008). 